\title{
Convergence analysis of accurate inverse Cholesky factorization
}

\author{
Yuka Yanagisawa ${ }^{1}$ and Takeshi Ogita ${ }^{1}$ \\ 1 Tokyo Woman's Christian University, 2-6-1 Zempukuji, Suginami-ku, Tokyo 167-8585, Japan \\ E-maild11m002@cis.twcu.ac.jp
}

Received October 30, 2012, Accepted November 11, 2012

\begin{abstract}
This paper is concerned with factorization of symmetric and positive definite matrices which are extremely ill-conditioned. Recently, Ogita and Oishi derived an iterative algorithm for an accurate inverse matrix factorization based on Cholesky factorization for such ill-conditioned matrices. We analyze the behavior of the algorithm in detail and explain its convergency by the use of numerical error analysis. Main analysis is that each iteration reduces the condition number of a preconditioned matrix by a factor around the relative rounding error unit until convergence, which is consistent with the existing numerical results.
\end{abstract}

Keywords convergence analysis, Cholesky factorization, ill-conditioned matrix, condition number, accurate numerical algorithm

Research Activity Group Quality of Computations

\section{Introduction}

Let $\mathbb{R}$ be the set of real numbers, and $\mathbb{F}$ a set of floating-point numbers conforming IEEE standard 754. Denote by $u$ the relative rounding error unit of floatingpoint arithmetic. In double precision (binary64) arithmetic, $u=2^{-53} \approx 1.1 \times 10^{-16}$. Throughout this paper, assume that neither overflow nor underflow occurs. For $A \in \mathbb{R}^{n \times n}$ define $\kappa(A):=\|A\|\left\|A^{-1}\right\|$ as the condition number of $A$, where $\|\cdot\|$ stands for spectral norm for matrices and Euclidean norm for vectors.

In this paper, we consider to treat the case where $A$ is symmetric, positive definite and extremely illconditioned such that

$$
\kappa(A) \gg u^{-1} .
$$

Let $A \in \mathbb{F}^{n \times n}$ with (1). When using Cholesky factorization to obtain a computed solution $\tilde{x}$ of $A x=b$ with $b \in \mathbb{F}^{n}$ by floating-point arithmetic, few or no correct digit can be expected for $\tilde{x}$. However, using OgitaOishi's algorithm [1], we can calculate a good approximate inverse $X$ of the exact Cholesky factor $\hat{R}$ such that $A=\hat{R}^{T} \hat{R}$ satisfying $\kappa\left(X A X^{T}\right) \approx 1$, and $A^{-1} \approx X X^{T}$. Thus, the Ogita-Oishi's algorithm can give an accurate numerical solution of $\tilde{x} \approx X X^{T} b$.

We analyze the behavior of the algorithm in detail and explain its convergency by the use of numerical error analysis. Main analysis to be explained is that each iteration of the Ogita-Oishi's algorithm reduces the condition number of a preconditioned matrix by a factor around $n^{2} u$ until convergence, which is consistent with the numerical results [1]. The key of the analysis is in multiplicative corrections of an approximate inverse $X$ of $\hat{R}$. The Ogita-Oishi's algorithm requires an algorithm for accurate dot product, which helps to treat extremely ill-conditioned matrices. Moreover, we show it is impor- tant to choose adequate computational precision for calculating dot products.

\section{Notation and definitions}

For $A=\left(a_{i j}\right), B=\left(b_{i j}\right) \in \mathbb{R}^{n \times n}$, we denote by $|A|=\left(\left|a_{i j}\right|\right) \in \mathbb{R}^{n \times n}$ a nonnegative matrix consisting of entrywise absolute values, and an inequality $A \leq B$ is understood entrywise, i.e., $a_{i j} \leq b_{i j}$ for all $(i, j)$. The notation $A \geq O$ means that all elements of $A$ are nonnegative. Similar notation applies to real vectors. Let $I$ denote the identity matrix.

The trace of $A=\left(a_{i j}\right) \in \mathbb{R}^{n \times n}$ is defined by

$$
\operatorname{tr}(A):=\sum_{i=1}^{n} a_{i i}
$$

The inversion of an upper triangular matrix $R \in \mathbb{F}^{n \times n}$ in solving a matrix equation $R T=I$ for $T$ using a standard numerical algorithm (e.g., xTRSV in BLAS and xTRTRI in LAPACK) is defined by

$$
T:=\operatorname{triinv}(R) .
$$

For readability we denote by $\varphi(\gamma)$ a constant such as $\varphi(\gamma)=c \cdot \gamma$ where $c:=\mathcal{O}(1)$ with $0<c \ll u^{-1}$.

Let $\mathbb{F}_{\Sigma}$ be a set of sum of floating-point numbers such that

$$
\mathbb{F}_{\Sigma}=\left\{x \in \mathbb{R}: x=\sum_{i=1}^{m} x_{i}, x_{i} \in \mathbb{F}, m \in \mathbb{N}\right\} .
$$

Note that $\mathbb{F} \subseteq \mathbb{F}_{\Sigma} \subset \mathbb{R}$.

Let $A, B \in \mathbb{F}_{\Sigma}^{n \times n}$. Assume that we have a function of calculating $C \in \mathbb{F}_{\Sigma}^{n \times n}$, for any $k, l \in \mathbb{N}, l \leq k$ satisfying

$$
|A B-C| \leq \varphi\left(u^{l}\right)|A B|+\varphi\left(u^{k}\right)|A||B| .
$$

Note that $C=\sum_{i=1}^{l} C_{i}$ with $C_{i} \in \mathbb{F}^{n \times n}, i=1,2, \ldots, l$. 
Namely, $C$ is an approximation of $A B$ as if computed in $k$-fold working precision and rounded into $l$ pieces of working precision floating-point numbers. We denote such a function as

$$
C_{1: l}=\{A B\}_{k}^{l}
$$

Such accurate dot product algorithms satisfying (2) have been proposed in [2-4].

Let $\left\langle A_{M}, A_{R}\right\rangle$ denote an interval matrix of the midpoint-radius representation such that

$$
\left\langle A_{M}, A_{R}\right\rangle:=\left\{X \in \mathbb{R}^{n \times n}:\left|X-A_{M}\right| \leq A_{R}\right\}
$$

with a midpoint $A_{M} \in \mathbb{F}^{n \times n}$ and a radius $A_{R} \in \mathbb{F}^{n \times n}$, $A_{R} \geq O$.

Similar notation in (3) applies to $B^{T} A B$, i.e.,

$$
\langle D, E\rangle=\left\{B^{T} A B\right\}_{k}^{1}
$$

which satisfies

$$
E \leq \varphi(u)\left|B^{T} A B\right|+\varphi\left(u^{k}\right)\left|B^{T}\right||A||B|
$$

for $D \in \mathbb{F}^{n \times n}$ with $D=D^{T}, E \in \mathbb{F}^{n \times n}, E \geq O$.

For later use, we define $\operatorname{shift}(A)$ for $A \in \mathbb{F}^{n \times n}$ by

$$
\operatorname{shift}(A):=c_{n} u \cdot \operatorname{tr}(A), \quad c_{n}=\frac{n+1}{1-(n+1)(n+2) u} .
$$

\section{Accurate and robust inverse Cholesky factorization}

Let $A=A^{T} \in \mathbb{F}^{n \times n}$ with $a_{i i}>0$ for $1 \leq i \leq n$. Suppose a standard numerical Cholesky factorization of $A$ runs to completion. Here "run to completion" means that no imaginary root appears in the factorization process. Throughout the paper, the Matlab-style notation $R=\operatorname{chol}(A)$ means a floating-point Cholesky factorization of $A$ using a standard numerical algorithm (e.g., xPOTRF in LAPACK) such that $A \approx R^{T} R$ where $R$ is an upper triangular matrix.

Suppose the exact Cholesky factorization of $A$ runs to completion such that $A=\hat{R}^{T} \hat{R}$. Then it holds that

$$
\kappa(\hat{R})=\kappa(A)^{\frac{1}{2}} \text {. }
$$

Let $\tilde{R}$ be a computed Cholesky factor of $A+\delta I \in$ $\mathbb{F}^{n \times n}$ for some suitable $\delta>0$, i.e., $\tilde{R}=\operatorname{chol}(A+\delta I)$. If $\kappa(A) \gtrsim u^{-1}$, then $\kappa(A+\delta I)$ is down to $\varphi\left(\alpha^{-1}\right)$ in almost all cases. Thus,

$$
\kappa(\tilde{R}) \approx \min \left(\kappa(A), \varphi\left(\alpha^{-1}\right)\right)^{\frac{1}{2}}
$$

follows. For more detail, see [1].

As long as $A$ is positive definite, $\operatorname{chol}(A+\delta I)$ never breaks down, even if taking the rounding errors into account [6]. For $\delta:=\operatorname{shift}(A)$, define $\tilde{\Delta}:=(A+\delta I)-\tilde{R^{T}} \tilde{R}$, then $\|\tilde{\Delta}\| \leq \delta$. For $n \ll u^{-1}, c_{n} \approx n$ and

$$
\|\tilde{\Delta}\| \approx n u \cdot \operatorname{tr}(A) \text {. }
$$

The following is an algorithm for an accurate inverse Cholesky factorization:

Algorithm 1 (Ogita-Oishi [1]) For a symmetric matrix $A=\left(a_{i j}\right) \in \mathbb{F}_{\Sigma}^{n \times n}$ with $a_{i i}>0$ for all $i$ and a specified tolerance $\varepsilon_{t o l} \leq 1$, the following algorithm calculates an upper triangular matrix $X_{1: m_{k}}^{(k)} \in \mathbb{F}_{\Sigma}^{n \times n}$ such that $\left\|X_{1: m_{k}}^{(k) T} A X_{1: m_{k}}^{(k)}-I\right\| \lesssim \varepsilon_{t o l}$.

$$
k=0, G^{(0)}:=A, E^{(0)}:=O, X_{1: 1}^{0}:=I
$$

repeat

$$
\begin{aligned}
& k=k+1 \\
& \delta_{k}:=\operatorname{shift}\left(G^{(k-1)}\right)+\left\|E^{(k-1)}\right\|
\end{aligned}
$$

Compute $S^{(k-1)} \in \mathbb{F}^{n \times n}$ with

$$
\begin{aligned}
& \left\{\begin{array}{l}
S_{i i}^{(k-1)} \geq G_{i i}^{(k-1)}+\delta_{k} \\
S_{i j}^{(k-1)}=G_{i j}^{(k-1)} \quad \text { for } i \neq j
\end{array} .\right. \\
& R^{(k)}:=\operatorname{chol}\left(S^{(k-1)}\right) \\
& T^{(k)}:=\operatorname{triinv}\left(R^{(k)}\right)
\end{aligned}
$$

$X_{1: m_{k}}^{(k)}:=\left\{X_{1: m_{k-1}}^{(k-1)} T^{(k)}\right\}_{m_{k}}^{m_{k}} \% m_{k}:=\left\lceil\frac{k}{2}\right\rceil+1$

$$
\left\langle G^{(k)}, E^{(k)}\right\rangle:=\left\{X_{1: m_{k}}^{(k) T} A X_{1: m_{k}}^{(k)}\right\}_{k+1}^{1}
$$

until $\left\|G^{(k)}-I\right\| \leq \varepsilon_{t o l}$

Remark 2 If it holds that $\left\|E^{(k-1)}\right\| \approx u\left\|G^{(k-1)}\right\|$, then

$$
\delta_{k}:=c_{n} u \cdot \operatorname{tr}\left(G^{(k-1)}\right)+\left\|E^{(k-1)}\right\| \approx n^{2} u\left\|G^{(k-1)}\right\| .
$$

If that is the case, then we have $\alpha \approx n^{2} u$. Note that $S^{(k-1)} \approx G^{(k-1)}+\delta_{k} I$, so that we use $G^{(k-1)}+\delta_{k} I$ instead of $S^{(k-1)}$ in the following analysis.

\section{Analysis}

We present the following lemmas for error bounds on an inversion of a triangular matrix.

Lemma 3 (Higham [5, Lemma 14.1]) Let $R \in$ $\mathbb{F}^{n \times n}$ be a triangular matrix. Let $X:=\operatorname{triinv}(R)$. Then

$$
|I-R X| \leq \varphi(u)|R||X| .
$$

Lemma 4 Let $R$ and $X$ be defined as in Lemma 3. Then

$$
\left\|I-X^{T} R^{T} R X\right\| \leq 2 \beta+\beta^{2}
$$

where $\beta:=\varphi(u)\||R\|X \mid\|$.

We omit the proof of Lemma 4 in this paper. If $\beta \lesssim 1$ satisfies, then $\beta^{2} \lesssim 1$ and

$$
\left\|I-X^{T} R^{T} R X\right\| \lesssim \varphi(u)\|R\|\|X\| .
$$

Next, we introduce a Rump's lemma for an estimation of a norm of a matrix.

Lemma 5 (Rump [7, Lemma 3.7]) Let a matrix $A \in \mathbb{R}^{n \times n}$ and a vector $x \in \mathbb{R}^{n}$ be given which are not correlated. Suppose $n \geq 4$. Then

$$
\|A\| \geq E\left(\frac{\|A x\|}{\|x\|}\right) \geq \frac{0.61}{(n-1)^{\frac{1}{2}}}\|A\|,
$$

where $E(\cdot)$ denotes an expectation value.

This implies $\|A\|\|x\| \approx\|A x\|$ in general.

Similarly to $[7$, Observation 3.6], we present the following observation.

Observation 6 Let $A=A^{T} \in \mathbb{F}^{n \times n}$ with $a_{i i}>0$ for $1 \leq i \leq n$. Let a triangular matrix $R \in \mathbb{F}^{n \times n}$ be a 
computed Cholesky factor of $A+\delta I$, i.e., $R=\operatorname{chol}(A+$ $\delta I)$ where $\delta:=\alpha\|A\|$ with $\alpha<1$. Let $T:=\operatorname{triinv}(R)$. Define $c:=\min \left(\varphi\left(\alpha^{-1}\right)^{1 / 2}, \kappa(R)\right)$. Then $\|T\| \approx c /\|R\|$.

Argument 7 If $A$ is not extremely ill-conditioned so that $\kappa(A) \lesssim \alpha^{-1}$, then $\kappa(R) \lesssim \alpha^{-1 / 2}$. Thus, $T$ is an approximate inverse of reasonable quality, so that $\|T\| \approx\left\|R^{-1}\right\|=c /\|R\|$. Suppose $A$ is extremely illconditioned. By $(6), \kappa(R)$ can be expected to be not much lager than $\varphi\left(\alpha^{-1}\right)^{1 / 2}$. Thus, we can expect in any case $\|T\| \approx c /\|R\|$.

The target of Analysis 8 is to confirm that $\| I-$ $X^{(1) T} A X^{(1)} \|$ cannot be much lager than 1 even for extremely ill-conditioned matrices. To explain this we utilize (8)-(11) for $k=1$ in Algorithm 1. Let $A \in \mathbb{F}^{n \times n}$ be given. For readability, $R^{(1)}$ and $X^{(1)}$ are abbreviated to $R$ and $X$, respectively.

Analysis 8 Define

$$
\begin{aligned}
& \Delta_{1}:=\left(A+\delta_{1} I\right)-R^{T} R, \\
& c_{\alpha}:=\min \left(\varphi\left(\alpha^{-1}\right), \kappa(A)\right) .
\end{aligned}
$$

We will analyze to show

$$
\begin{aligned}
& \|X\| \approx\left(\frac{c_{\alpha}}{\|A\|}\right)^{\frac{1}{2}}, \\
& \left\|I-X^{T} A X\right\| \approx \alpha c_{\alpha}, \\
& \left\|X^{T} A X\right\| \approx 1 .
\end{aligned}
$$

Argument 9 First, we estimate $\|X\|$ in (16). If $A$ is not extremely ill-conditioned so that $\kappa\left(A+\delta_{1} I\right)=$ $\kappa(A)<\alpha$, then $X$ is an approximate inverse of reasonable quality, so that $\|X\| \approx\left\|R^{-1}\right\|=(\kappa(A) /\|A\|)^{1 / 2}$. Now suppose $A$ is extremely ill-conditioned. On (9), from (8), we have

$$
\left\|A+\delta_{1} I\right\| \approx\|A\|+\alpha\|A\| .
$$

From (7), it holds

$$
\left\|\Delta_{1}\right\| \approx \alpha\|A\|
$$

Combining (14), (19) and (20) yields

$$
\|R\| \approx\|A\|^{\frac{1}{2}} .
$$

By Observation 6 and (21), we have

$$
\|X\| \approx \frac{c_{\alpha}^{\frac{1}{2}}}{\|R\|} \approx\left(\frac{c_{\alpha}}{\|A\|}\right)^{\frac{1}{2}},
$$

which explains (16).

Next, we estimate $\left\|I-X^{T} A X\right\|$ in (17). It holds

$$
\begin{aligned}
& \left\|I-X^{T} A X\right\| \\
& \quad \leq\left\|I-X^{T} R^{T} R X\right\|+\left\|X^{T}\left(R^{T} R-A\right) X\right\| .
\end{aligned}
$$

From (16), we have $\|A\|^{1 / 2}\|X\| \approx c_{\alpha}^{1 / 2}$, and $\varphi(u)$. $\||R||X|\| \lesssim 1$. Therefore, applying (13) yields

$$
\left\|I-X^{T} R^{T} R X\right\| \approx \varphi(u)\|R\|\|X\| .
$$

Moreover, using (8) and (9), we have

$$
\left\|X^{T}\left(R^{T} R-A\right) X\right\| \leq\left\|X^{T}\right\|\left\|\Delta_{1}-\delta I\right\|\|X\|
$$

$$
\begin{aligned}
& \lesssim\left\|X^{T}\right\|(\alpha\|A\|+\alpha\|A\|)\|X\| \\
& \approx \alpha\|R\|^{2}\|X\|^{2} .
\end{aligned}
$$

Inserting (24) and (25) into (23) implies

$$
\left\|I-X^{T} A X\right\| \approx \varphi(u)\|R\|\|X\|+\alpha\|R\|^{2}\|X\|^{2} \approx \alpha c_{\alpha},
$$

which explains (17).

The definition of $c_{\alpha}$ implies $\left\|I-X^{T} A X\right\| \lesssim 1$, so that (18) follows.

The target of Analysis 10 is to explain that the condition number of a preconditioned matrix eventually converges to 1 after some iterations. Suppose we are at the $k$-th iteration. By numerical results [1], we observe

$$
\kappa\left(X^{(k) T} A X^{(k)}\right) \approx 1+\varphi\left(\alpha^{k}\right) \kappa(A) .
$$

To explain this we utilize the estimates in Analysis 8. For readability, $X_{1: m_{k}}^{(k)}$ and $X_{1: m_{k+1}}^{(k+1)}$ are abbreviated to $X$ and $X^{\prime}$, respectively.

\section{Analysis 10 Define}

$$
\begin{aligned}
\delta_{k} & :=\operatorname{shift}(G), \\
X & :=\operatorname{triinv}(R), \\
\Delta_{2} & :=G-X^{T} A X, \\
\Delta_{3} & :=\left(G+\delta_{k} I\right)-R^{\prime} R^{\prime}, \\
T & :=\operatorname{triinv}\left(R^{\prime}\right), \\
\Delta_{4} & :=X^{\prime}-X T, \\
c_{\alpha} & :=\min \left(\varphi\left(\alpha^{-1}\right), \kappa\left(X^{T} A X\right)\right) .
\end{aligned}
$$

Assume

$$
\begin{aligned}
& \|X\| \approx\left(\frac{\alpha^{-k}}{\|A\|}\right)^{\frac{1}{2}}, \\
& \left\|X^{T} A X\right\| \approx 1, \\
& \kappa\left(X^{T} A X\right) \approx \alpha^{k} \kappa(A) .
\end{aligned}
$$

Then we will analyze

$$
\begin{aligned}
& \left\|X^{\prime}\right\| \approx\left(\frac{c_{\alpha} \alpha^{-k}}{\|A\|}\right)^{\frac{1}{2}}, \\
& \left\|X^{\prime T} A X^{\prime}\right\| \approx 1, \\
& \kappa\left(X^{\prime} T A X^{\prime}\right) \approx c_{\alpha}^{-1} \alpha^{k} \kappa(A) .
\end{aligned}
$$

Argument 11 First, we estimate $\left\|X^{\prime}\right\|$ in (34). On (27), the computation of $X^{T} A X$ by $\left\{X^{T} A X\right\}_{k+1}^{1}$ implies

$$
\left\|\Delta_{2}\right\| \approx u\left\|X^{T} A X\right\|+u^{k+1}\left\|X^{T}\right\|\|A\|\|X\| \approx u .
$$

Therefore with (32)

$$
\|G\| \approx\left\|X^{T} A X\right\|+\left\|\Delta_{2}\right\| \approx 1+u \approx 1 .
$$

On (28), using a similar way from (19) to (21), we have

$$
\left\|\Delta_{3}\right\| \approx \alpha\|G\| \approx \alpha
$$

and

$$
\left\|R^{\prime}\right\| \approx\|G\|^{\frac{1}{2}}
$$


Hence Observation 6 yields

$$
\|T\| \approx \frac{c_{\alpha}^{\frac{1}{2}}}{\left\|R^{\prime}\right\|} \approx\left(\frac{c_{\alpha}}{\|G\|}\right)^{\frac{1}{2}} \approx c_{\alpha}^{\frac{1}{2}} .
$$

Therefore, the computation of $X^{\prime}$ in $m_{k}$-fold working precision gives

$$
\begin{aligned}
\left\|\Delta_{4}\right\| & \approx u^{\left\lceil\frac{k}{2}\right\rceil+1}\|X T\|+u^{\left\lceil\frac{k}{2}\right\rceil+1}\|X\|\|T\| \\
& \approx u^{\left\lceil\frac{k}{2}\right\rceil+1}\left(\frac{c_{\alpha} \alpha^{-k}}{\|A\|}\right)^{\frac{1}{2}}
\end{aligned}
$$

and with (31) and (41) it follows

$$
\left\|X^{\prime}\right\| \approx\|X T\| \approx\|X\|\|T\|+\left\|\Delta_{4}\right\| \approx\left(\frac{c_{\alpha} \alpha^{-k}}{\|A\|}\right)^{\frac{1}{2}} .
$$

Next we estimate $\left\|I-X^{\prime} A X^{\prime}\right\|$. Then

$$
\begin{aligned}
\| I- & X^{\prime} A X^{\prime} \| \\
= & \left\|I-\left(X T+\Delta_{4}\right)^{T} A\left(X T+\Delta_{4}\right)\right\| \\
\leq & \left\|I-T^{T} X^{T} A X T\right\|+2\left\|T^{T} X^{T} A \Delta_{4}\right\| \\
& \quad+\left\|\Delta_{4}^{T} A \Delta_{4}\right\| .
\end{aligned}
$$

Here

$$
\begin{aligned}
\| I- & T^{T} X^{T} A X T \| \\
= & \left\|\left(I-T^{T} G T\right)-T^{T} \Delta_{2} T\right\| \\
= & \left\|\left(I-T^{T}\left(R^{\prime} R^{\prime}+\Delta_{3}-\delta_{k} I\right) T\right)-T^{T} \Delta_{2} T\right\| \\
\leq & \left\|I-T^{T} R^{\prime} R^{\prime} T\right\|+\left\|T^{T} \Delta_{3} T\right\|+\delta_{k}\left\|T^{T} T\right\| \\
& \quad+\left\|T^{T} \Delta_{2} T\right\| .
\end{aligned}
$$

From (41), it holds $\|T\|\left\|R^{\prime}\right\| \approx c_{\alpha}^{1 / 2}$, and $\varphi(u)\left\||T|\left|R^{\prime}\right|\right\| \lesssim 1$. Therefore, applying (13) yields

$$
\left\|I-T^{T} R^{\prime T} R^{\prime} T\right\| \lesssim \varphi(u)\|T\|\left\|R^{\prime}\right\| \approx \varphi(u) c_{\alpha}^{\frac{1}{2}} .
$$

Moreover, by (37)-(39) and (41), we have

$$
\begin{aligned}
& \left\|T^{T} \Delta_{3} T\right\| \leq\|T\|^{2}\left\|\Delta_{3}\right\| \approx \alpha c_{\alpha}, \\
& \delta_{k}\left\|T^{T} T\right\|=\delta_{k}\|T\|^{2} \approx \alpha c_{\alpha}\|G\| \approx \alpha c_{\alpha},
\end{aligned}
$$

and

$$
\left\|T^{T} \Delta_{2} T\right\| \leq\|T\|^{2}\left\|\Delta_{2}\right\| \approx u c_{\alpha} .
$$

Inserting (46)-(49) into (45) implies

$$
\begin{aligned}
\left\|I-T^{T} X^{T} A X T\right\| & \approx \varphi(u) c_{\alpha}^{\frac{1}{2}}+\alpha c_{\alpha}+\alpha c_{\alpha}+u c_{\alpha} \\
& \approx \alpha c_{\alpha} .
\end{aligned}
$$

We estimate $\left\|T^{T} X^{T} A \Delta_{4}\right\|$ and $\left\|\Delta_{4}^{T} A \Delta_{4}\right\|$ in (44). Using (37), (38), (41) and (42) yields

$$
\begin{aligned}
\left\|T^{T} X^{T} A \Delta_{4}\right\| & =\left\|T^{T} X^{T} A X X^{-1} \Delta_{4}\right\| \\
& =\left\|T^{T}\left(G-\Delta_{2}\right) X^{-1} \Delta_{4}\right\| \\
& \leq\|T\|\left(\|G\|+\left\|\Delta_{2}\right\|\right)\left\|X^{-1}\right\|\left\|\Delta_{4}\right\| \\
& \lesssim u^{\left\lceil\frac{k}{2}\right\rceil+1} c_{\alpha} \alpha^{-\frac{k}{2}} .
\end{aligned}
$$

Moreover, by (42), it holds

$$
\left\|\Delta_{4}^{T} A \Delta_{4}\right\|=\left\|\Delta_{4}\right\|^{2}\|A\| \approx u^{k+2} c_{\alpha} \alpha^{-k} .
$$

Inserting (50)-(52) into (44) implies

$$
\begin{aligned}
\left\|I-X^{\prime T} A X^{\prime}\right\| & \approx \alpha c_{\alpha}+u^{\left\lceil\frac{k}{2}\right\rceil+1} c_{\alpha} \alpha^{-\frac{k}{2}}+u^{k+2} c_{\alpha} \alpha^{-k} \\
& \approx \alpha c_{\alpha} .
\end{aligned}
$$

The definition of $c_{\alpha}$ implies $\left\|I-X^{T} A X\right\| \lesssim 1$, so that (35) follows.

To see (36) suppose first that $X$ is not extremely illconditioned, from (27) and (37), we have $\kappa\left(X^{T} A X\right)<$ $\alpha^{-1}$. Thus, the definition (30) implies $c_{\alpha}=\kappa\left(X^{T} A X\right)$. By $(53), \kappa\left(X^{\prime} T A X^{\prime}\right) \approx 1 \approx c_{\alpha}^{-1} \kappa\left(X^{T} A X\right) \approx$ $c_{\alpha}^{-1} \alpha^{k} \kappa(A)$ using (33). Second, suppose $X$ is extremely ill-conditioned, the definition (30) implies $c_{\alpha}=\varphi\left(\alpha^{-1}\right)$. Denote the $\nu$-th row of $X^{\prime}$ by $x^{(\nu)}, 1 \leq \nu \leq n$ such that $\left\|X^{\prime}\right\| \approx\left\|x^{(\nu)}\right\|$. Then

$$
\left\|x^{(\nu)}\left(\hat{R} X^{\prime}\right)^{-1}\right\|=\left\|e_{\nu} \hat{R}^{-1}\right\| \approx\left\|\hat{R}^{-1}\right\|,
$$

where $e_{\nu}$ denotes the $\nu$-th row of the identity matrix. Since $X$ is extremely ill-conditioned, we may assume at least one row $x^{(\nu)}$ to be sufficiently independent of $\left(\hat{R} X^{\prime}\right)^{-1}$, so that Lemma 5 yields

$$
\left\|\left(\hat{R} X^{\prime}\right)^{-1}\right\| \approx \frac{\left\|x^{(\nu)}\left(\hat{R} X^{\prime}\right)^{-1}\right\|}{\left\|x^{(\nu)}\right\|} .
$$

Inserting (34) and (54) into (55) implies

$$
\left\|\left(\hat{R} X^{\prime}\right)^{-1}\right\| \approx \frac{\left\|\hat{R}^{-1}\right\|}{\left\|X^{\prime}\right\|} \approx c_{\alpha}^{-\frac{1}{2}} \alpha^{\frac{k}{2}} \kappa(\hat{R}) .
$$

Therefore, from (5), (35) and (56), we have

$$
\kappa\left(X^{\prime} A X^{\prime}\right) \approx 1 \cdot\left\|\left(\hat{R} X^{\prime}\right)^{-1}\right\|^{2} \approx c_{\alpha}^{-1} \alpha^{k} \kappa(A)
$$

which explains (36).

\section{References}

[1] T. Ogita and S. Oishi, Accurate and robust inverse Cholesky factorization, Nonlinear Theory and Its Applications, IEICE, 3 (2012), 103-111.

[2] T. Ogita, S. M. Rump and S. Oishi, Accurate sum and dot product, SIAM J. Sci. Comput., 26 (2005), 1955-1988.

[3] S. M. Rump, T. Ogita and S. Oishi, Accurate floating-point summation part I: faithful rounding, SIAM J. Sci. Comput., 31 (2008), 189-224.

[4] S. M. Rump, T. Ogita and S. Oishi, Accurate floating-point summation part II: sign, K-fold faithful and rounding to nearest, SIAM J. Sci. Comput., 31 (2008), 1269-1302.

[5] N. J. Higham, Accuracy and Stability of Numerical Algorithms, 2nd ed., SIAM, Philadelphia, PA, 2002.

[6] S. M. Rump, Verification of positive definiteness, BIT, 46 (2006), 433-452.

[7] S. M. Rump, Inversion of extremely ill-conditioned matrices in floating-point, Jpn J. Indust. Appl. Math., 26 (2009), 249277 . 